\title{
Epidemiologia do consumo de medicamentos em crianças de centro urbano da região sul do Brasil*
}

\author{
Epidemiology of consumption of medicines by children of an urban population in \\ the southern of Brazil
}

\author{
Jorge U. Béria**, Cesar G. Victora**, Fernando C. Barros"*, Ana B. Teixeira**, Cintia Lombardi**
}

\begin{abstract}
BERIA, J. U. et al. Epidemiologia do consumo de medicamentos em crianças de centro urbano da região sul do Brasil. Rev. Saúde Pública, 27: 95-104, 1993. Foram investigados os padrões do consumo de medicamentos em uma coorte de 4.746 crianças de Pelotas, RS, Brasil e as influências de variáveis socioeconômicas, biológicas e de utilização de serviços de saúde. $O$ delineamento foi transversal aninhado em estudo longitudinal e o período inves tigado foi 15 dias. O consumo global alcançou $56 \%$ das crianças, sendo mais de $50 \%$ em todas as classes sociais. Os medicamentos mais utilizados foram ácido acetil salicílico, vitaminas com sais minerais, associações antigripais, mebendazole e estimulantes do apetite. Mais de 60,0\% dos medicamentos eram indicados por médicos (inclusive dipirona estimulantes do apetite). Os principais motivos do consumo foram gripe, febre e falta de apetite. Ser primogênito foi fator de risco para o consumo. As crianças com pouco apetite na semana anterior consumiam duas vezes mais do que aquelas com bom apetite. É preocupante o alto consumo de aspirina, principalmente devido à associação desse produto com a Síndrome de Reye em crianças. Outro ponto a ser questionado a respeito é a mensagem que talvez inadvertida ou inconscientemente possa estar sendo passada a essas crianças: o consumo de medicamentos é uma rotina e a resposta para qualquer problema. Nesse sentido, parece que se estará preparando o terreno para a dependência de medicamentos e drogas ilícitas.
\end{abstract}

Descritores: Hábitos de consumo de medicamentos. Saúde infantil.

\section{Introdução}

Com o acentuado avanço da ciência e da tecnologia em nosso tempo, os medicamentos foram crescendo em seu papel, chegando a constituir um dos principais remédios utilizados para mitigar os muitos tipos de dor a que estamos sujeitos ${ }^{8}$.

Os medicamentos transformaram-se, também, em importante mercadoria, movimentando altas cifras anualmente. Em 1985, o mercado de medicamentos movimentou 43 bilhões de dólares. As desigualdades são enormes: $75 \%$ da população mundial (países pobres) consumiram so-

\footnotetext{
* $\quad$ Parte de Tese de Doutorado apresentada por J.U.Béria à Universidade Federal do Rio Grande do Sul, em 1991. Pesquisa realizada com auxílio financeiro do Intemational Development Research Center (Canadá), Overseas Development Administration (Reino Unido), Divisão de Saúde Familiar da Organização Mundial da Saúde e Conselho Nacional de Desenvolvimento Científico e Tecnológico.

** Departamento de Medicina Social da Faculdade de Medicina da Universidade Federal de Pelotas - Pelotas, RS - Brasil.
}

Separatas/Reprints: J.U.Béria - Caixa Postal 464-96001-970 Pelotas, RS - Brasil. mente $21 \%$ dos medicamentos utilizados naquele ano. O Brasil, em 1985, era o décimo mercado de medicamentos do mundo, com vendas de 1,4 bilhōes de dólares, representando $1,5 \%$ do mercado mundial. As indústrias farmacêuticas multinacionais dominam $78 \%$ do mercado brasileiro de medicamentos ${ }^{30}$.

Quando todo esse aparato científico, tecnológico e econômico é acionado na expectativa de aliviar o sofrimento das crianças, podemos imaginar a complexidade de fatores envolvidos.

São escassos os estudos recentes de base populacional sobre o tema. Em geral, apontam para um alto consumo entre crianças e um menor consumo de medicamentos entre os grupos sociais de baixa renda, sendo a maioria dos medicamentos consumidos com indicação médica. Em revisão bibliográfica nos últimos dez anos encontramos apenas três estudos no Brasil com amostra representativa probabilística (Ribeirão Preto e Araraquara em São Paulo e Nova Iguaçu no Rio), nenhum com ênfase específica para o consumo em crianças ${ }^{3,7,24}$.

Outro fator relevante do presente estudo é a utilização do conceito de classe social como categoria explicativa. Os indicadores tradicionais de estratificação, como renda e escolaridade, mesmo nas tentativas de sua utilização a partir de uma 
perspectiva dialética, configuram grupos humanos que não compartilham necessariamente a mesma situação de classe, já que implicam cortes arbitrários do pesquisador que não refletem a complexidade da estrutura social ${ }^{5,6}$. É importante lembrar, ainda, as dificuldades em obter dados fidedignos sobre renda.

O consumo de medicamentos é, também, um indicador importante para avaliar a qualidade dos serviços de saúde e a efetividade da propaganda de medicamentos junto aos médicos e à população ${ }^{13}$.

A idéia central deste estudo é desvendar alguns determinantes do consumo de medicamentos, suas inter-relaçס̋es e as magnitudes de suas influências em uma coorte de 4.746 crianças. Pretende, também, fornecer material para os planejadores de saúde e formadores de recursos humanos do setor, no sentido de orientar as políticas e currículos relacionados ao tema.

\section{Metodologia}

A presente investigação, realizada em 1986 em Pelotas, RS (217.000 habitantes) ${ }^{20}$, tem um delineamento híbrido, sendo um estudo transversal alinhado em um estudo longitudinal ${ }^{11}$. São utilizadas variáveis coletadas em diferentes fases da coorte.

\section{$O$ estuda longitudinal das crianças nascidas em 1982}

Este estudo, até 1990 , havia se desdobrado em quatro fases, incluindo o estudo perinatal e três acompanhamentos da coorte de 6.011 crianças urbanas nascidas em hospitais (mais de $99 \%$ dos nascimentos totais). $O$ estudo foi planejado para avaliar a influência sobre a saúde infantil de uma série de fatores perinatais, demográficos, ambientais, alimentares e assistenciais, dentro de um quadro mais amplo definido pela estrutura social 27,28 .

No terceiro acompanhamento, realizado em 1986, foi estudado o consumo de medicamentos. Foi realizado um censo da cidade sendo visitados 77.199 domicílios e encontradas 4.746 crianças que correspondiam a $84 \%$ da coorte de nascidos em hospitais de Pelotas em 1982. A idade das crianças neste acompanhamento variava entre $35 \mathrm{e}$ 53 meses. O trabalho de campo foi realizado de dezembro de 1985 a abril de 1986 . (O presente artigo referir-se-á a 1986, para simplificar).

Em relação ao consumo de medicamentos, as perguntas formuladas às mães ou responsáveis, em 1986, foram as seguintes:

"A criança recebeu algum remédio nos últimos 15 dias, inclusive remédio para febre ou vitaminas?"
"Qual é?"

"Para tratar o quê?"

"Quem receitou?"

"Foi tomado regularmente por um mês ou mais?"

Os entrevistadores solicitavam às mães que mostrassem, sempre que disponíveis, os medicamentos que estavam sendo consumidos. No questionário havia espaço para identificação de até cinco medicamentos. As informações mais detalhadas descritas acima foram colhidas para os primeiros três medicamentos referidos, devido a resultados de outro estudo mostrar que foi rara a utilização de mais de três medicamentos no período estudado ${ }^{3}$. Posteriormente ficou confirmado que apenas 2,7\% das crianças haviam consumido mais de três medicamentos.

Adotou-se a definição de medicamento da Organização Mundial da Saúde (OMS) 17: "Medicamento $e$ toda substância contida em um produto farmacêutico, utilizada para modificar ou investigar sistemas fisiológicos ou estados patológicos, em benefício da pessoa em que se administra". A homeopatia pode não se enquadrar totalmente nesta definição, mas foi também considerada no presente estudo. Os medicamentos foram classificados conjuntamente por dois dos autores (JUB e ABT).

As variáveis estudadas foram as seguintes:

\section{Variável dependente}

Consumo de medicamentos - percentual de crianças utilizando medicamentos nos 15 dias anteriores à entrevista.

Variáveis independentes

Classe social - Tomando por base alguns indicadores socioeconômicos da pessoa de maior renda da família (tipo de ocupação, posição em relação aos meios de produção, setor e ramo de atividade, renda mensal, tipo de estabelecimento se autônomo; número de empregados - se empregador - e escolaridade) foi possível classificar as famílias em classes sociais ou frações de classes ${ }^{6}$.

Renda - Quintis de renda familiar per capita mensal em 1986, em salários mínimos.

Estado nutricional da criança - escores $\mathrm{z}$ para peso/idade e altura/idade ${ }^{29}$. A criança era pesada com uma balança CMS tipo Salter, especialmente projetada para pesquisa domiciliar e medida em pé, sendo utilizado um antropômetro desenvolvido pela própria equipe de pesquisa. Em função de seu peso, altura, idade e sexo, cada criança foi classificada em escores $\mathrm{z}$ em relação ao padrão do "National Center for Health Statistics" dos EUA ${ }^{16}$. Assim, foram consideradas como desnutridas as 
crianças que estivessem com um escore $\mathrm{z}$ menor do que a média menos dois desvios-padrão e, como eutróficas, as demais.

Outras variáveis independentes estudadas foram: escolaridade da mãe, sexo da criança, ordem de nascimento, idade da criança, amamentação ao seio, apetite da criança (na semana anterior), consultas médicas da criança (nos três meses anteriores à entrevista), hospitalizaçōes da criança (no ano anterior). As variáveis independentes incluíram algumas informaçōes coletadas por ocasião do nascimento das crianças, no inicio do estudo longitudinal (ordem de nascimento) e outras coletadas durante os acompanhamentos. Do segundo acompanhamento, em 1984, utilizamos a variável educação da mãe e do terceiro, as demais.

A análise estatística das informações seguiu os seguintes passos: análise univariada com a descrição da distribuição das variáveis dependente e independentes na população estudada, utilizando o programa estatístico SPSS/PC+; análise bivariada constando do cruzamento da variável dependente com as independentes através de tabelas de contingência (teste qui-quadrado) utilizando o programa SPSS/PC+, e teste para tendência linear em proporçőes '; por último, análise multivariada com a investigação do efeito conjunto de variáveis independentes sobre a variável dependente através de regressão logística incondicional, utilizando o programa estatístico EGRET.

As estimativas fornecidas pela análise estatística podem ser expressas como razão de produtos cruzados (RPC) com o respectivo intervalo de confiança de $95 \%$ e o teste de razão de verossimilhanças (TRV) categórico e para tendência linear. A RPC pode ser expressa de duas maneiras: a) não ajustada ou bruta e b) controlando para as variáveis cujo efeito se deseja neutralizar. A RPC expressa a magnitude das influências das variáveis independentes sobre a variável dependente. A RPC para eventos considerados raros (prevalência menor do que $10 \%$ ) é quase equivalente à razão de prevalências (RP).

No presente estudo, a variável dependente possui prevalência elevada (56\%). Neste caso, para facilitar a interpretação dos resultados, se fez necessário converter a RPC em RP. A fórmula utilizada foi:

$$
\begin{gathered}
R P=\frac{R P C}{1+P \tilde{\exp }(\mathrm{RPC}-1)} \\
\text { Pñ } \exp =\text { Prevalência na população não exposta. }
\end{gathered}
$$

A análise multivariada foi realizada conforme modelo teórico hierarquizado expresso na Figura 1 e que contempla as variáveis independentes associadas ao consumo de medicamentos no presente estudo. Assim, os efeitos das variáveis sociais

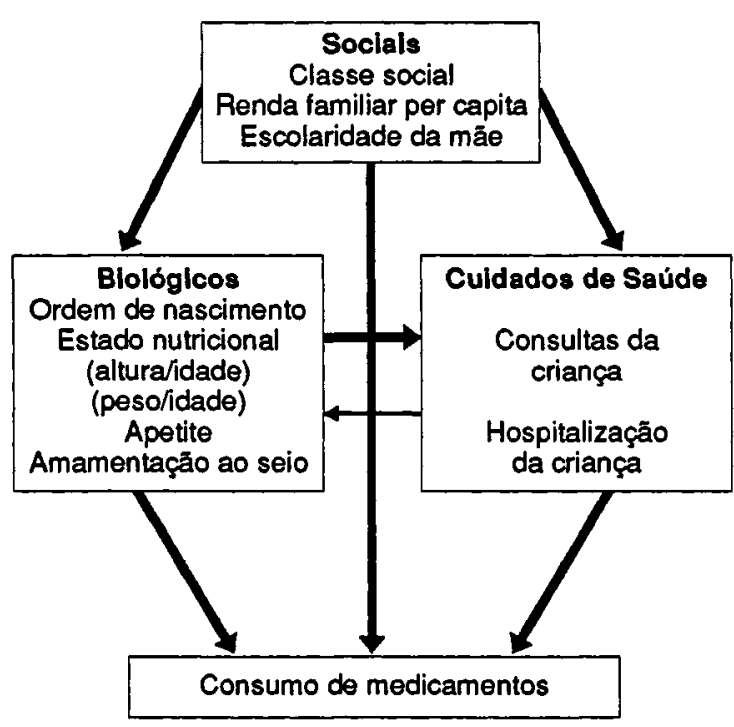

Figura 1. Modelo teórico hierarquizado dos fatores associados ao consumo de medicamentos de crianças nascidas em 1982, Pelotas, RS, 1986.

(classe social, renda familiar per capita e educação da mãe) não foram ajustados para nenhuma das outras variáveis em estudo, pois no modelo teórico utilizado, o social determina o biológico. Os efeitos das variáveis biológicas (ordem de nascimento, altura/idade, peso/idade, apetite e amamentação ao seio) foram ajustados para as variáveis sociais. Finalmente, os coeficientes das variáveis relativas aos cuidados de saúde (consultas e internaçðes da criança) foram ajustados para as variáveis sociais e biológicas. Conforme o modelo teórico, o efeito dos cuidados de saúde sobre os fatores biológicos seria pequeno, não sendo portanto realizado o ajuste neste sentido. É essencial salientar que este modelo não contempla um importante determinante que é a morbidade da criança. Conforme será salientado em maiores detalhes na discussão, esta variável não foi investigada no presente estudo.

\section{Resultados}

Das 4.746 crianças localizadas, $55,8 \%$ haviam consumido algum medicamento nos 15 dias que precederam a entrevista. Entre estas, o número total de medicamentos consumidos foi de 4.322 , sendo que $31,3 \%$ das crianças haviam utilizado um medicamento e $8,8 \%$, três ou mais. O consumo crônico de medicamentos por um mês ou mais atingiu $9,5 \%$ das crianças. Vinte e quatro por cento das crianças consumiram medicamentos com três ou mais fármacos. Os médicos foram responsáveis pela prescrição de $62,7 \%$ dos medicamentos, seguidos pelas mães $(32,3 \%$ ) (Tabela 1 ). 
Tabela 1. Distribuição das crianças nascidas em 1982 segundo consumo de medicamentos num periodo de 15 dias, Pelotas, RS, 1986.

\begin{tabular}{|c|c|c|}
\hline & $\%$ & $N$ \\
\hline Consumo de medicamentos & & 4.746 \\
\hline $\begin{array}{l}\text { Não consumiu } \\
\text { Consumiu }\end{array}$ & $\begin{array}{l}44,2 \\
55,8\end{array}$ & \\
\hline Número de medicamentos consumidos & & 4.746 \\
\hline $\begin{array}{l}\text { Nenhum } \\
\text { Um medicamento } \\
\text { Dois medicamentos } \\
\text { Três medicamentos ou mais }\end{array}$ & $\begin{array}{r}44,2 \\
31,3 \\
15,7 \\
8,8\end{array}$ & \\
\hline $\begin{array}{l}\text { Consumo de medicamentos conforme } \\
\text { número de fármacos }\end{array}$ & & 4.746 \\
\hline $\begin{array}{l}\text { Três ou mais fármacos }\left({ }^{*}\right) \\
\text { Um ou dois fármacos } \\
\text { Não consumiu }\end{array}$ & $\begin{array}{l}23,6 \\
32,2 \\
44,2\end{array}$ & \\
\hline $\begin{array}{l}\text { Consumo de medicamentos por um } \\
\text { mês ou mais }\end{array}$ & & 4.746 \\
\hline $\begin{array}{l}\text { Consumiu } \\
\text { Não consumiu }\end{array}$ & $\begin{array}{r}9,5 \\
90,5\end{array}$ & \\
\hline Quem indicou os medicamentos("*) & & 4.176 \\
\hline $\begin{array}{l}\text { Médico } \\
\text { Māe } \\
\text { Parentes e vizinhos } \\
\text { Balconistas de farmácias } \\
\text { Pai } \\
\text { Outros profissionais de saúde } \\
\text { Religiosos }\end{array}$ & $\begin{array}{r}62,5 \\
32,5 \\
2,1 \\
1,7 \\
0,8 \\
0,2 \\
0,1\end{array}$ & \\
\hline
\end{tabular}

(*) Excluidos os reidrantes orais.

(*) Número de medicamentos consumidos com informação de quem indicou.

Os produtos mais consumidos (Tabela 2) foram o ácido acetil salicílico $(24,7 \%)$, as combinações de vitaminas mais sais minerais $(9,5 \%) \mathrm{e}$, em terceiro lugar, as associaçðes antigripais $(8,9 \%)$. Foi surpreendente o número de estimulantes do apetite, que apareceram em quinto lugar $(4,5 \%)$. Esta tabela, também mostra o percentual de cada produto indicado por médicos. O ácido acetil salicílico correspondia a quase um quarto dos medicamentos utilizados, sendo $30,5 \%$ com indicação médica. Há uma tendência dos medicamentos mais utilizados apresentarem menor índice de indicação médica.

Os problemas de saúde citados mais freqüentemente como motivos da utilização de medicamentos foram gripe (17,3\%), febre (14,9\%) e falta de apetite $(12,1 \%)$ (Figura 2). Surgiram também alguns motivos pouco usuais para o consumo, como por exemplo, analgésico (AAS) ministrado "depois do banho para prevenir gripe", "porque a
Tabela 2. Distribuição percentual dos medicamentos de maior consumo em crianças nascidas em 1982 e indicaçāo médica, Pelotas, RS, 1986.

\begin{tabular}{lrcc}
\hline Medicamentos & \multicolumn{2}{c}{ Consumo } & $\begin{array}{c}\text { Indicação } \\
\text { Médica } \\
(\%)\end{array}$ \\
\cline { 2 - 3 } & Número Percentual & Pun \\
\hline Acido Acetil Salicilico & 1.069 & 24,7 & 30,5 \\
Vitaminas + Sais minerais & 412 & 9,5 & 55,4 \\
Associaçoses antigripais & 384 & $\mathbf{8 , 9}$ & 50,0 \\
Mebendazole & 221 & 5,1 & 88,0 \\
Estimulantes do apetite & 195 & 4,5 & 73,2 \\
Reidradantes orais & 165 & 3,8 & 70,5 \\
Polivitaminas & 110 & 2,5 & 87,0 \\
Teofilina & 105 & 2,4 & 89,3 \\
Sulfametoxasol + & & & \\
Trimetropim & 83 & 1,9 & 87,7 \\
Terbutalina & 77 & 1,8 & 91,8 \\
Fenoximetilpenicilina & 75 & 1,7 & 87,5 \\
Sulfato Ferroso & 72 & 1,7 & 91,4 \\
Homeopatia & 63 & 1,5 & 62,9 \\
Dipirona & 60 & 1,4 & 74,0 \\
Complexo B & 60 & 1,4 & 84,5 \\
Acetaminofen & 58 & 1,3 & 64,2 \\
Outros medicamentos & 1.112 & 25,8 & 76,3 \\
\hline Total de medicamentos & 4.322 & 99,9 & 62,5 \\
\hline
\end{tabular}

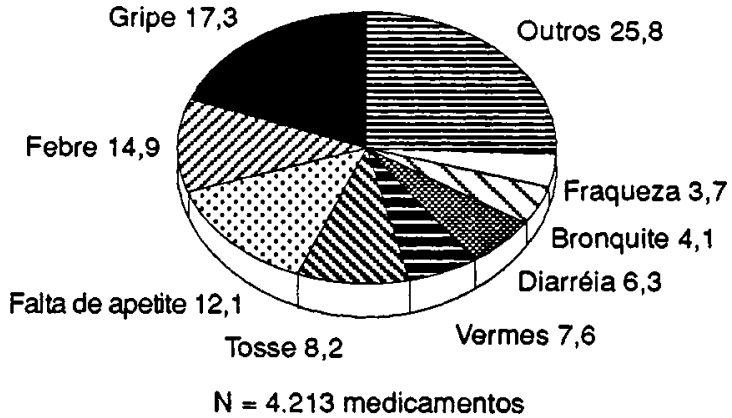

Figura 2. Principais motivos de consumo de medicamentos em crianças nascidas em 1982, Pelotas, RS, 1986.

criança tomou chuva", "porque o irmão tomou", "a criança pediu", "toda vez que chora", entre outros.

A seguir descrevemos as distribuiçōes das variáveis independentes utilizadas no estudo. Em relação à classe social, as crianças pertenciam em grande parte ao proletariado não típico $(42 \%)$. Quanto à renda familiar mensal, a maioria das famílias estava na faixa de menos de um salário mínimo (aproximadamente 50 dólares) per capita. Sessenta e dois por cento das mães apresentavam de quatro a dez anos de escolaridade. Do sexo masculino eram $51 \%$ das crianças, sendo que $39 \%$ eram o primeiro filho do casal. A idade variava entre 35 e 53 meses. Em relação à amamentação ao seio, $39 \%$ das crianças foram amamentadas menos de três meses, enquanto que $18 \%$ mamaram por um ano ou mais. Em $29 \%$ dos casos, as mães rela- 
tavam pouco apetite da criança na semana anterior à entrevista. $\mathrm{O}$ estado nutricional, medido pelo escore $\mathrm{z}$, apontou $8,5 \%$ de desnutridos segundo 0 critério altura-idade e apenas $3 \%$ conforme 0 peso-idade. Quanto à utilização dos serviços de saúde, em $53 \%$ das crianças houve o relato de uma ou mais consultas médicas nos três meses anteriores à entrevista, e $9 \%$ das crianças haviam sido hospitalizadas no periodo de um ano.

Antes de iniciar a análise multivariada propriamente dita, foi realizada uma análise bivariada, cruzando os fatores socioeconômicos com o consumo de medicamentos. Os resultados são expressos como razão de produtos cruzados, os quais podem ser transformados em razão de prevalências. Não houve associação entre o consumo global de medicamentos e classe social. As diferenças significantes apareceram apenas quando os principais grupos terapêuticos foram discriminados. Assim, a nova pequena burguesia e a burguesia foram as classes onde as crianças mais consumiam analgésicos e antiinflamatórios, e medicamentos para o aparelho respiratório enquanto que antiparasitários e medicamentos para o aparelho digestivo eram mais consumidos por crianças do subproletariado e proletariado típico (Fig. 3). Por outro lado, a RPC do consumo de medicamentos conforme a renda fa-

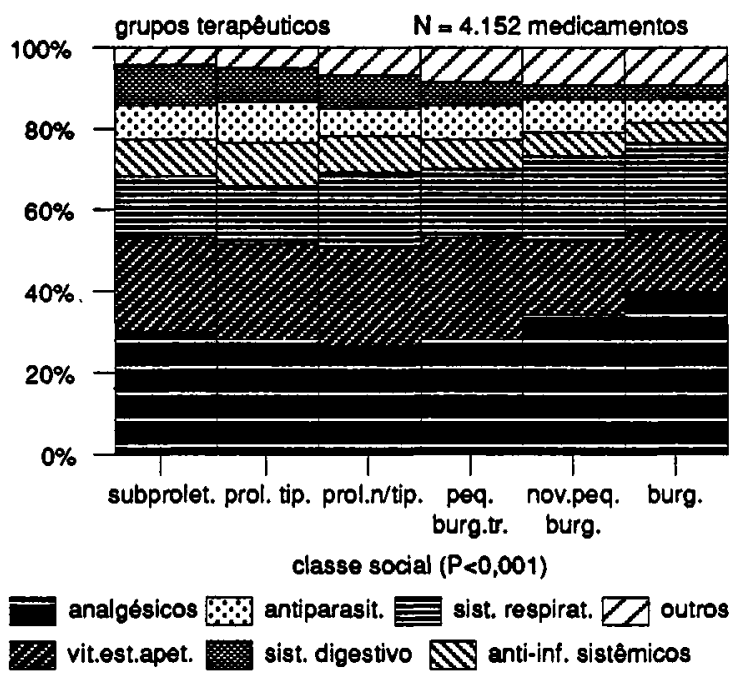

Flgura 3. Distribuição percentual dos medicamentos consumidos pelas crianças nascidas em 1982 contorme os principais grupos terapêuticos, de acordo com classe social, Pelotas, RS, 1986.

Tabela 3. Razão de produtos cruzados do consumo de medicamentos em crianças nascidas em 1982 segundo algumas variáveis sociais, Pelotas, RS, 1986.

\begin{tabular}{|c|c|c|c|c|}
\hline \multirow[t]{2}{*}{ Variáveis Independentes } & \multirow{2}{*}{$\begin{array}{l}\text { Razão de produtos cruzados } \\
\text { não ajustada e intervalos de } \\
\text { confiança de } 95 \%\end{array}$} & \multirow{2}{*}{$\begin{array}{l}\text { Número } \\
\text { de crianças }\end{array}$} & \multicolumn{2}{|c|}{ TRV } \\
\hline & & & categórico & tendência \\
\hline Classe social & & & $\begin{array}{c}11,93 \\
(p=0,06)\end{array}$ & NSA \\
\hline $\begin{array}{l}\text { Subproletariado } \\
\text { Proletariado típico } \\
\text { Proletariado não típico } \\
\text { Pequena burguesia }\end{array}$ & $\begin{array}{l}1,00 \\
0,96(0,77-1,18) \\
1,20(0,98-1,47)\end{array}$ & $\begin{array}{r}468 \\
1.239 \\
1.975\end{array}$ & & \\
\hline $\begin{array}{l}\text { tradicional } \\
\text { Nova pequena burguesia } \\
\text { Burguesia }\end{array}$ & $\begin{array}{l}1,19(0,93-1,51) \\
1,05(0,78-1,41) \\
1,15(0,66-2,00)\end{array}$ & $\begin{array}{r}651 \\
287 \\
58\end{array}$ & & \\
\hline $\begin{array}{l}\text { Quintis de renda familiar } \\
\text { p/capita em SMS }\end{array}$ & & & $\begin{array}{c}17,51 \\
(p<0,005)\end{array}$ & $\begin{array}{c}10,56 \\
(p<0,005)\end{array}$ \\
\hline $\begin{array}{l}1(<=0,28) \\
2(0,29-0,46) \\
3(0,47-0,74) \\
4(0,75-1,38) \\
5(1,39-31,30)\end{array}$ & $\begin{array}{l}1,00 \\
1,25(1 ; 05-1,50) \\
1,39(1,16-1,67) \\
1,38(1,15-1,65) \\
1,33(1,10-1,60)\end{array}$ & $\begin{array}{l}956 \\
958 \\
966 \\
948 \\
918\end{array}$ & & \\
\hline $\begin{array}{l}\text { Escolariedade da mãe em } \\
\text { anos }\end{array}$ & & & $\begin{array}{c}26,25 \\
(p<0,001)\end{array}$ & $\begin{array}{c}12,02 \\
(p<0,001)\end{array}$ \\
\hline $\begin{array}{l}0 \\
1-3 \\
4-5 \\
6-10 \\
11-22\end{array}$ & $\begin{array}{l}1,22(0,90-1,64) \\
1,00 \\
1,38(1,16-1,65) \\
1,50(1,26-1,79) \\
1,58(1,29-1,93)\end{array}$ & $\begin{array}{r}229 \\
741 \\
1.478 \\
1.469 \\
829\end{array}$ & & \\
\hline
\end{tabular}

TRV - Teste de razão de verossimilhanças.

NSA - Não se aplica.

SMS - Salários Mínimos. 
miliar per capita mostra que as crianças classificadas nos quintis três e quatro apresentavam um consumo de medicamentos $17 \%$ mais alto do que as do quintil um (RPC $=1,4$ transformada em RP equivale aproximadamente a um excesso de $17 \%$ ). As crianças de mães com escolaridade acima de seis anos consumiam $20 \%$ ( $R P C=1,5$ ) mais medicamentos do que aquelas cujas mães haviam estudado menos de três anos (Tabela 3 ). Como em relação à escolaridade materna havia dúvida a respeito da influência que poderia exercer no consumo, independente da inserção de classe, foi realizada análise controlando para classe social e renda familiar. Os resultados foram praticamente os mesmos.

A Tabela 4 revela que os primogênitos consomem mais medicamentos que seus irmãos, mesmo controlando para classe social, renda familiar e escolaridade da mãe. Ter irmãos mais velhos foi um fator de proteção em relação ao consumo de medicamentos. Há uma tendência linear significante e após ajustar para as variáveis sociais, os segundos filhos consumiram 7\% ( $R C P=0,86)$ menos medicamentos que os primogênitos e as crianças com dois ou mais irmãos mais velhos consumiram $12 \%$ ( $R P C=0,76$ ) menos (Fig. 4). Dois outros fatores biológicos estudados - sexo e idade - não estavam associados ao consumo de medicamentos.

No modelo teórico, os fatores de confusão foram definidos como as variáveis hierarquicamente superiores ao fator de risco em estudo. No entanto, é também possível incluir no modelo multivariado, variáveis hierarquicamente inferiores que re-

Tabela 4. Razāo de produtos cruzados do consumo de medicamentos em crianças nascidas em 1982 segundo algumas variáveis biológicas, Pelotas, RS, 1986.

\begin{tabular}{|c|c|c|c|}
\hline Variáveis Independentes & $\begin{array}{l}\text { Razão de produtos cruzados } \\
\text { não ajustada e intervalos de } \\
\text { confiança de } 95 \%\end{array}$ & $\begin{array}{l}\text { Controlando para classe social } \\
\text { renda familiar e educ. da mãe } \\
\text { (intervalos de confiança de } 95 \% \text { ) }\end{array}$ & $\begin{array}{l}\text { Número } \\
\text { de } \\
\text { crianças }\end{array}$ \\
\hline \multicolumn{4}{|l|}{ Ordem de nascimento } \\
\hline $\begin{array}{l}\text { Primogênito } \\
\text { Segundo } \\
\text { Terceiro ou + } \\
\text { TRV (2 GL) } \\
\text { TRV (tendência) }\end{array}$ & $\begin{array}{l}1,00 \\
0,84(0,73-0,97) \\
0,71(0,62-0,81) \\
25,06(p<0,001) \\
25,05(p<0,001)\end{array}$ & $\begin{array}{l}1,00 \\
0,86(0,74-0,99) \\
0,76(0,66-0,88) \\
14,28(p<0,001) \\
14,77(p<0,001)\end{array}$ & $\begin{array}{l}1.843 \\
1.346 \\
1.557\end{array}$ \\
\hline \multicolumn{4}{|l|}{$\begin{array}{l}\text { Amamentação ao seio em } \\
\text { meses }\end{array}$} \\
\hline $\begin{array}{l}\text { Nunca mamou } \\
<3 \\
3-5 \\
6-11 \\
12+ \\
\operatorname{TRV}(4 \mathrm{GL})\end{array}$ & $\begin{array}{l}1,00 \\
1,19(0,96-1,49) \\
1,25(0,99-1,59) \\
0,99(0,77-1,28) \\
0,91(0,71-1,15) \\
17,77(p<0,001)\end{array}$ & $\begin{array}{l}1,00 \\
1,16(0,93-1,45) \\
1,20(0,95-1,53) \\
0,95(0,73-1,22) \\
0,91(0,71-1,16) \\
14,77(p<0,01)\end{array}$ & $\begin{array}{r}377 \\
1.835 \\
1.028 \\
672 \\
834\end{array}$ \\
\hline \multicolumn{4}{|l|}{ Estado nutricional } \\
\hline \multicolumn{4}{|l|}{ Peso/idade } \\
\hline $\begin{array}{l}\text { Adequado } \\
\text { Desnutrido ( }) \\
\text { TRV (1GL) }\end{array}$ & $\begin{array}{l}1,00 \\
1,64(1,17-2,30) \\
8,74(p<0,005)\end{array}$ & $\begin{array}{l}1,00 \\
1,78(1,27-2,51) \\
11,68(p<0,001)\end{array}$ & $\begin{array}{r}4.588 \\
158\end{array}$ \\
\hline \multicolumn{4}{|l|}{ Altura/idade } \\
\hline $\begin{array}{l}\text { Adequado } \\
\text { Desnutrido }(t) \\
\text { TRV (1GL) }\end{array}$ & $\begin{array}{l}1,00 \\
1,38(1,12-1,70) \\
9,19(p<0,005)\end{array}$ & $\begin{array}{l}1,00 \\
1,47(1,19-1,81) \\
12,69(p<0,001)\end{array}$ & $\begin{array}{r}4.342 \\
404\end{array}$ \\
\hline \multicolumn{4}{|l|}{ Apetite na última semana } \\
\hline $\begin{array}{l}\text { Bom } \\
\text { Pouco } \\
\text { TRV (1 GL) }\end{array}$ & $\begin{array}{l}1,00 \\
2,07(1,81-2,36) \\
119,97(p<0,001)\end{array}$ & $\begin{array}{l}1,00 \\
2,12(1,85-2,42) \\
125,47(p<0,001)\end{array}$ & $\begin{array}{l}3.391 \\
1.355\end{array}$ \\
\hline
\end{tabular}

TRV - Teste de razão de verossimilhanças.

GL - Graus de liberdade.

$f$ - Menor que a média menos dois desvios-padrão (NCHS) ${ }^{16}$. 


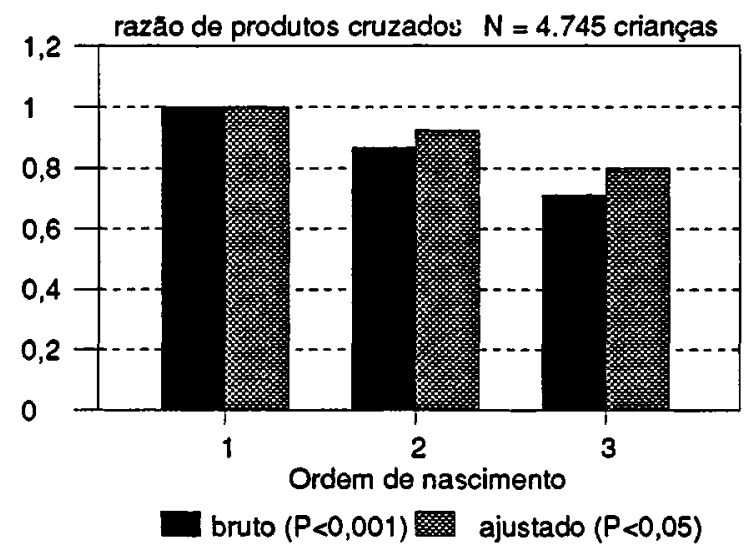

Figura 4. Razáo de produtos cruzados do consumo de medicamentos $e$ ordem de nascimento de crianças nascidas em 1982, Pelotas, RS, 1986

presentam prováveis mecanismos da ação do fator em estudo. Por exemplo, pode-se ajustar o efeito de ordem de nascimento para o número de consultas, investigando assim até que ponto consultas mais frequientes seriam responsáveis pelo maior consumo de medicamentos dos primogênitos. Se isto fosse verdadeiro, as RPCs para as diferentes categorias de ordem de nascimento tenderiam à unidade. Esta análise foi realizada, mas as RPCs permaneceram praticamente as mesmas, sigerindo que o efeito da ordem de nascimento não é mediado pelo número de consultas.

A Tabela 4 também mostra que as crianças amamentadas ao seio por um ano ou mais consu- miam $4 \%$ menos medicamentos do que as que nunca mamaram. Entretanto, os intervalos de confiança incluíram a unidade.

Quanto ao estado nutricional, as crianças desnutridas conforme o critério peso/idade consumiam $25 \%(\mathrm{RPC}=1,64)$ mais medicamentos que as com peso/idade adequado. Controlando para variáveis sociais, este valor subia para $30 \%(\mathrm{RPC}=1,78$ ). Neste caso, classe social, renda e escolaridade materna configuram um exemplo de fatores de confusão negativos ${ }^{4}$. Como os desnutridos pertencem a famílias de classe social, renda familiar e escolaridade materna menos favorecidas, seria esperado que consumissem menos. Mesmo assim, na análise bruta, consomem mais medicamentos. Quando os fatores sociais são ajustados, os desnutridos aparecem consumindo ainda mais medicamentos, pois o efeito de confusão das variáveis sociais foi eliminado 21 . Em relação à altura/idade os valores eram $16 \%(R P C=1,38)$ e $19 \%(R P C=1,47)$, respectivamente.

As crianças com pouco apetite na última semana consumiam duas vezes mais medicamentos do que aquelas com bom apetite. Controlando para variáveis sociais, o valor permanecia aproximadamente o nesmo.

A Tabela 5 mostra os resultados relativos à utilização cos serviços de saúde. $\mathrm{Na}$ análise multivariada, estes resultados foram ajustados para as variáveis sociais e biológicas conforme o modelo teórico hierarquizado referido na metodologia. As crianças que consultaram uma vez os serviços de saúde nos três meses anteriores à entrevista, con-

Tabela 5. Razão de produtos cruzados do consumo de medicamentos em crianças nascidas em 1982 segundo algumas variáveis de utilização de serviços de saúde, Pelotas, RS, 1986.

\begin{tabular}{|c|c|c|c|}
\hline Variáveis Independentes & $\begin{array}{l}\text { Razão de produtos cruzados } \\
\text { não ajustada } \theta \text { intervalos de } \\
\text { confiança de } 95 \%\end{array}$ & $\begin{array}{l}\text { Controlando para variáveis } \\
\text { scciais e biológicas (") (inter- } \\
\text { valos de confiança de } 95 \% \text { ) }\end{array}$ & $\begin{array}{l}\text { Número de } \\
\text { crianças }\end{array}$ \\
\hline \multicolumn{4}{|l|}{$\begin{array}{l}\text { Consultas nos últimos irês } \\
\text { meses }\end{array}$} \\
\hline $\begin{array}{l}\text { Nenhuma } \\
\text { Uma } \\
\text { Duas } \\
\text { Três } \\
\text { Quatro ou + } \\
\text { TRV (4 GL) } \\
\text { TRV (tendência) }\end{array}$ & $\begin{array}{l}1,00 \\
1,80(1,58-2,05) \\
3,09(2,51-3,79) \\
3,60(2,70-4,79) \\
4,40(2,95-6,56) \\
243,20(p<0,001) \\
231,80(p<0,001)\end{array}$ & $\begin{array}{l}1,00 \\
1,71(1,50-1,96) \\
2,92(2,36-3,61) \\
3,46(2,59-4,64) \\
4,25(2,83-6,38) \\
210,37(p<0,001) \\
202,45(p<0,001)\end{array}$ & $\begin{array}{r}2.215 \\
1.581 \\
528 \\
274 \\
148\end{array}$ \\
\hline \multicolumn{4}{|l|}{ Internações no último ano } \\
\hline $\begin{array}{l}\text { Nenhuma } \\
\text { Uma ou mais } \\
\text { TRV (1 GL) }\end{array}$ & $\begin{array}{l}1,00 \\
1,29(1,05-1,58) \\
5,99(p<0,05)\end{array}$ & $\begin{array}{l}1,00 \\
1,33(1,08-1,65) \\
7,15(p<0,01)\end{array}$ & $\begin{array}{r}4.324 \\
422\end{array}$ \\
\hline $\begin{array}{l}\text { TRV - Teste de razão d } \\
\text { GL - Graus de liberda } \\
\text { (") - Classe social, re }^{*} \text { - tacäo peso/idade }\end{array}$ & $\begin{array}{l}\text { ossimilhanças. } \\
\text { familiar per capita, educ } \\
\text { ura/idade e apetite. }\end{array}$ & da mãe, ordem de $n$ & \\
\hline
\end{tabular}


sumiam $31 \%(\mathrm{RPC}=1,8)$ mais medicamentos do que as que não consultaram. $O$ consumo aumentou linearmente com o número de consultas, sendo que as crianças que consultaram quatro vezes ou mais consumiam duas $(R P C=4,4)$ vezes mais medicamentos do que aquelas que não consultaram. As crianças que foram hospitalizadas no ano anterior consumiram $12 \%(\mathrm{RPC}=1,3)$ mais do que as que não internaram. Controlando para variáveis de confusão, estes valores permaneciam praticamente os mesmos.

\section{Discussão}

O estudo longitudinal das crianças nascidas em Pelotas em 1982 demonstrou que, apesar das dificuldades inerentes à pesquisa epidemiológica no Terceiro Mundo, foi possível identificar uma coorte de crianças, representativa de toda a população de uma cidade de tamanho médio, e acompanhá-la prospectivamente com uma perda relativamente pequena por um período aproximado de quatro anos.

Alguns problemas e limitações do estudo de consumo de medicamentos podem ser levantados:

1) A idade das crianças ser de uma amplitude limitada, pois todas haviam nascido em 1982 e tinham no momento do estudo entre três e quatro anos e meio, aproximadamente. Existem referências na literatura a um maior consumo de medicamentos nos dois primeiros anos de vida ${ }^{2,9,12}$, portanto nesta investigação estaríamos perdendo o período de maior consumo nas crianças.

2) Perdas de acompanhamento - O percentual de crianças localizadas esteve sempre acima de $80 \%$, e as diferenças entre as crianças localizadas e as não localizadas foram pequenas ${ }^{28}$.

3) Viés de memória - As mães ou responsáveis eram solicitados a referir os medicamentos consumidos nos quinze dias anteriores à entrevista. Embora a maioria dos estudos revisados tenham utilizado esse mesmo periodo de tempo, existe relato na literatura de que o ideal seria perguntar sobre o consumo no dia anterior à entrevista $^{26} \circ$ que diminuiria este tipo de erro, mas ao mesmo tempo prejudicaria a comparação com os resultados de outros estudos.

4) A proporção do orçamento familiar destinada a compra de medicamentos para a criança não foi avaliada devido a não haver informação sobre onde os medicamentos foram adquiridos, se em farmácia comercial ou obtidos gratuitamente.

5) A indicação médica dos medicamentos poderia ter sido melhor estudada. Não foi colhida informação sobre repetição de receitas anteriores, isto é, se o medicamento foi indicado pelo médico para o problema atual ou se foi repetição de uma prescrição anterior.

6) Não foi realizado levantamento da morbidade no período estudado devido às dificuldades operacionais de tais inquéritos. Assim, não se pode conhecer que percentual dos problemas de saúde referidos pela população em estudo são tratados com medicamentos. Apenas havia a informação dos problemas para os quais os medicamentos estavam sendo utilizados.

Em relação ao consumo de medicamentos, é notável o fato de que mais da metade das crianças havia consumido um ou mais medicamentos nos 15 dias anteriores à entrevista. Este nível de consumo foi superior aos achados de Barros ${ }^{3}$, em Ribeirão Preto, para menores de cinco anos, onde alcançou $32,1 \%$, mas inferior ao observado em um estudo norte-americano para crianças de três a seis anos $(69,6 \%)^{12}$. No estudo realizado em Araraqua$\mathrm{ra}, \mathrm{SP}^{24}$, o consumo entre os menores de cinco anos chegou a $50 \%$ e, como no presente estudo, a maioria dos medicamentos tinha indicação médica.

É preocupante este alto consumo de medicamentos em crianças de uma idade na qual não existem grandes riscos para a saúde. Isso mostra que há uma grande disponibilidade de medicamentos nos domicílios e, portanto, risco das crianças terem acesso a esses produtos, sem a orientação dos responsáveis, e sofrerem intoxicações.

Outro ponto a ser questionado é a mensagem que talvez, inadvertida ou inconscientemente, possa estar sendo passada a essas crianças: o consumo de medicamentos é uma rotina e a resposta para qualquer problema. Nesse sentido, não se estaria preparando o terreno para a dependência de medicamentos e drogas ilícitas? ${ }^{10}$ Os principais beneficiados com a presente situação seriam a indústria e o comércio de medicamentos, além dos interessados no subjacente controle social advindo desse tipo de prática.

No que se refere à classe social, é incoerente que a nova pequena burguesia e burguesia consumam mais analgésicos e medicamentos para o aparelho respiratório, quando sabemos que a morbidade das crianças da coorte foi maior nas classes desprivilegiadas ${ }^{28}$. As variaçбes no uso destas duas categorias medicamentosas estão possivelmente mais associadas a distorçōes terapêuticas do que a diferenças epidemiológicas. No estudo de Ribeirão Preto ${ }^{3}$ foi encontrado que as frações de classe com maior prevalência de morbidade eram as que apresentavam menor consumo de medicamentos. Os antiparasitários (anti-helmínticos principalmente) e medicamentos para o aparelho digestivo (reidratante oral, antidiarréicos, e outros) 
foram utilizados com maior frequiência nas classes desprivilegiadas, o que reflete a distribuição social da incidência desses problemas.

Outro achado do estudo foi o maior consumo de medicamentos entre os primogênitos (apesar da magnitude ser discreta). A ansiedade e insegurança da familia talvez estejam entre os fatores determinantes desse comportamento. É importante que os serviços de saúde estejam atentos para esse tipo de conduta e reforcem a rede de apoio familiar no sentido de baixar a ansiedade inerente a uma experiência tão nova e, por vezes, angustiante como a de ser "pais de primeira viagem".

É preocupante, também, o alto consumo de aspirina, principalmente devido à associação desse produto com a Síndrome de Reye (uma encefalopatia aguda associada com degeneração gordurosa do fígado) em crianças com doenças virais. Apesar da raridade desta síndrome (incidência anual inferior a um caso por 10.000 crianças), as autoridades sanitárias de diversos países - como os Estados Unidos e a Inglaterra - passaram a desaconselhar o uso de aspirina em menores de doze anos, particularmente na presença de febre, infecções respiratórias ou varicela ${ }^{18,19}$. Entre nossas crianças que utilizaram aspirina, $50 \%$ o fizeram devido a febre, $35,8 \%$ por infecçð̃es respiratórias e $0,2 \%$ por varicela. O acetaminofen e, recentemente, o ibuprofeno ${ }^{14}$ têm sido recomendados como alternativas para crianças com febre. Como são drogas mais caras do que a aspirina e não estão disponiveis nos postos de saúde (ibuprofeno somente em apresentação para adultos), é possível que - no caso de restrições à aspirina - a população de baixa renda passe a utilizar como alternativa outros produtos perigosos. Este é o caso da dipirona (medicamento proibido em muitos países desenvolvidos) que apesar de seu uso estar associado a agranulocitose é distribuída gratuitamente pela Central de Medicamentos em postos de saúde e hospitais, além de estar disponível em farmácias, bares e armazéns ${ }^{15}$. Tanto a dipirona quanto os estimulantes do apetite (considerados medicamentos inadequados) $)^{10,23,25}$, em sua grande maioria, foram indicados por médicos.

$O$ uso racional de medicamentos permanece uma área não priorizada pelos governos do Terceiro Mundo. Como inexistem bons sistemas de informação na maioria dos países, reações adversas não são monitorizadas e a educação continuada não é implementada. O Brasil tem um longo caminho a percorrer no que se refere ao uso racional dos medicamentos. Segundo Rozenfeld 22 , "é urgente e inadiável a construção de uma nova cultura dos medicamentos, centrada no estudo sistemático dos impactos biológicos e sociais dos tratamentos médicos e na divulgação ampla dos resultados desses estudos. $O$ objetivo dessa nova cultura deve ser a transformação do medicamento de mercadoria em instrumento para a preservação e restauração da saúde".

\section{Agradecimento}

A Sharon R. A. Huttly (Universidade de Londres) pelo auxílio na análise estatística.

BÉRIA, J. U, et al. [Epidemiology of consumption of medicines by children of an urban population in the south of Brazill]. Rev. Saúde Pública, 27: 95-104, 1993. The consumption of medicines among a populationbased cohort of 4,746 children born in 1982 in Pelotas, Brazil, was studied when the children were aged $3-4,5$ years. Fifty six percent of the mothers reported that their children had taken one or more medicines during a two-week period; $29.5 \%$ of the products were fixed combinations of three or more components, (which was taken as an indicator of poor quality). Almost $10 \%$ of the children had used a given medicine for one month or more. Aspirin, combinations of vitamins and mineral supplements and cough and cold combinations were the medicines most frequently used. The commonest reasons for taking medicines were colds, fevers and lack of appetite. The latter was the commonest reason for longterm use and also for that of combinations. Physicians' prescriptions were responsible for more than $60 \%$ of the medicines used (including dipyrone and appetite stimulants). In all social classes the consumption was above $50 \%$. Children classified in the fifth quintile of family income consumed $14 \%$ more medicines than those in the first quintile. Children with two or more older siblings consumed $12 \%$ less medicines than the elder ones. Malnourished children, according to weight for age, consumed $30 \%$ more medicines than the wellnourished. Children consulting a doctor four times or more during the three-month period before the interview were using two times more medicines than children who had had no consultation during the same period. The frequent use of aspirin is a reason for concern as it has been associated with Reye's syndrome in children. It is also important to stress the danger of poisoning resulting from medicines available at home. Another noteworthy aspect concerns the messages transmitted to the children regarding the use of medicines for almost every conceivable reason which could possibly lead to medicine or illicit drug addiction.

Keywords: Drug use habits. Child health.

\section{Referências Bibliográficas}

1. ARMITAGE, P. \& BERRY, G. Slatistical methods in medical research. 2nd ed. Oxford, Blackwell, 1987.

2. BARROS, F. C. \& VICTORA, C. G. Avaliação do manejo de diarréia em menores de cinco anos no Nordeste do Brasil. J. Pediat., 65: 420-59, 1989. 
3. BARROS, M. B. A. Saúde e classe social: um estudo sobre morbidade e consumo de medicamentos. Ribeirāo Preto, 1983. [Tese de Doutoramento - Faculdade de Medicina de Ribeirão Preto-USP].

4. BÉRIA, J. U. Epidemiologia do consumo de medicamentos em crianças de Pelotas, RS, Brasil: remédio não é brinquedo. Porto Alegre, 1991. [Tese de Doutoramento Faculdade de Medicina da UFRGS]

5. BRESLOW, N. E. \& DAY, N. E. Statistical methods in cancer research: (The analysis of case-control studies. Lyon, IARC, 1980. v. 1) (IARC Scient. Publ., 32).

6. BRONFMAN, M; LOMBARDI, C.; FACCHINI, L. A.; VICTORA, C. G.; BARROS, F. C.; BERIA, J. U.; TELXEIRA, A. M. B. Operacionalização do conceito de classe social em estudos epidemiologicos. Rev. Saúde Pública, 22: 253-65, 1988.

7. CORDEIRO, H. A. A indústria da saúde no Brasil. Rio de Janeiro, Graal, 1980.

8. GIGANTE, A. G. $O$ que é remédio. São Paulo, Brasiliense, 1988.

9. HAAK, H. Padrões de consumo de medicamentos em dois povoados da Bahia (Brasil). Rev. Saúde Pública, 23: 143-51, 1989.

10. INTERNATIONAL AGRANULOCYTOSIS AND APLASTIC ANEMIA STUDY. Risks of agranulocytosis and aplastic anemia: a first report of their relation to drug use with special reference to analgesics. JAMA, 256: 1749-57, 1986.

11. KLEINBAUM, D. G.; KUPPER, L. L.; MORGENSTERN, H. Epidemiologic research. New York, VNR, 1982.

12. KOVAR, M. G. Use of medication and vitamin-mineral suplements by children and youths. Public Health Rep., 100: 470-3, 1985.

13. LAPORTE, J. R.; TOGNONI, G.; ROZENFELD, S. Epidemiologia do medicamento: princípios gerais. São Paulo, Hucitec-Abrasco, 1989.

14. MANAGEMENT of childhood fever. [Editorial]. Lancet, 338: 1049-50, 1991.

15. MINISTÉRIO DA SAUDE. O médico e a famácia básica. Brasília, CEME, 1987.

16. NATIONAL CENTER FOR HEALTH STATISTICS. NCHS growth curves for children, birth-18 years. Vital Health Statist. Ser. 11 (165) 1977.

17. ORGANIZACIÓN MUNDIAL DE LA SALUD. Comite de Expertos en uso de medicamentos esenciales, Gine- bra, 1984. Informe. Ginebra, 1985. (OMS-Ser. Inf. Tec., 722).

18. PINSKY, P. F.; HURWITZ, E. S.; SCHONBERGER, L. B.; GUNN, W. J. Reye's syndrome and aspirin: evidence for dose-response effect. JAMA, 260:657-61, 1988.

19. REYE'S syndrome and the giving of aspirin to children. [Editorial]. Lancet, 1: 1396, 1986.

20. ROSA, M. Geografia de Pelotas. Pelotas. Ed. da Ufpel, 1986.

21. ROTHMAN, K. J. Modern epidemiology. Boston, Little \& Brown, 1986.

22. ROZENFELD, S. Avaliaçẫo do uso dos medicamentos como estratégia para a reorientação da política de insumos em saúde. Cad. Saúde Pública, 4: 388-402, 1989.

23. SILVERMAN, M.: LEE, P. R.; LYDECKER, M. Drug promotion: the third world revisited. Int. J. Health Serv., 16: 659-67, 1986.

24. SIMÕES, M. J. S. \& FARACHE FILHO, A. Consumo de Medicamentos em região do Estado de São Paulo (Brasil), 1985. Rev. Saúde Pública, 22: 494-9, 1988.

25. VANCE, M. A. \& MILLINGTON, W. R. Principles of irrational drug therapy. Int. J. Health Serv., 16: 355-61, 1986.

26. VAN DER GEEST, S. \& HARDON, A. Drugs use: methodological suggestions for field research in developing countries. Heallh Policy Plan., 2: 152-8, 1988.

27. VICTORA, C. G.; BARROS, F. C.; MARTINES, J. C.; BERIA, J. U.; VAUGHAN, J. P. Estudo longitudinal das crianças nascidas em 1982 em Pelotas, RS. Brasil. Metodologia e resultados preliminares. Rev. Saúde Pública, 19: 58-68, 1985.

28. VICTORA, C. G.; BARROS, F. C.; VAUGHAN, J. P. Epidemiologia da desigualdade. 2a. ed. São Paulo, Hucitec, 1988.

29. WATERLOW, J. C.; BUZINA, R.; KELLER, W.; LANE, J. M.; NICHAMAN, M. Z.; TANNER, J. M. The presentation and use of height and weight data for comparing the nutritional status of children under the age of 10 years. Bull. Wld. Health Organ., 55: 489-98, 1977.

30. WORLD HEALTH ORGANIZATION. The world drug situation. Geneva, 1988.

Recebido para publicaçäo em 31.3 .1992 Reapresentado em 16.11.1992 Aprovado para publicação em 13.1.1993 